\title{
Determinants, Norms, and the Spread of Circulant Matrices with Tribonacci and Generalized Lucas Numbers
}

\author{
Juan Li, ${ }^{1,2}$ Zhaolin Jiang, ${ }^{1}$ and Fuliang $\mathrm{Lu}^{1}$ \\ ${ }^{1}$ Department of Mathematics, Linyi University, Linyi, Shandong 276000, China \\ ${ }^{2}$ Department of Mathematics, Shandong Normal University, Jinan, Shandong 250014, China
}

Correspondence should be addressed to Zhaolin Jiang; jzh1208@sina.com

Received 28 March 2014; Accepted 26 April 2014; Published 11 May 2014

Academic Editor: Tongxing Li

Copyright (C) 2014 Juan Li et al. This is an open access article distributed under the Creative Commons Attribution License, which permits unrestricted use, distribution, and reproduction in any medium, provided the original work is properly cited.

Circulant matrices play an important role in solving ordinary and partial differential equations. In this paper, by using the inverse factorization of polynomial of degree $n$, the explicit determinants of circulant and left circulant matrix involving Tribonacci numbers or generalized Lucas numbers are expressed in terms of Tribonacci numbers and generalized Lucas numbers only. Furthermore, four kinds of norms and bounds for the spread of these matrices are given, respectively.

\section{Introduction}

Circulant matrices have important applications in solving various partial differential equations. By the radial properties of the fundamental solution and radial symmetric of the solution domain, Chen et al. [1] showed the circulant or block circulant features of the coefficient matrices for problems under pure Dirichlet or Neumann boundary condition. Lei and Sun [2] proposed the preconditioned CGNR (PCGNR) method with a circulant preconditioner to solve such Toeplitz-like systems. Using circulant matrix, Karasozen and Simsek [3] considered periodic boundary conditions such that no additional boundary terms will appear after semidiscretization. In [4], a semicirculant preconditioner applied to a problem, subject to Dirichlet boundary conditions at the inflow boundaries, was examined. In [5], the resulting dense linear system exhibits so much structure that it can be solved very efficiently by a circulant preconditioned conjugate gradient method. A method was described for obtaining finite difference approximation solutions of multidimensional partial differential equations satisfying boundary conditions specified on irregularly shaped boundaries by using circulant matrices and fast Fourier transform (FFT) convolutions in [6]. Brockett and Willems [7] showed how the important problems of linear system theory can be solved concisely for a particular class of linear systems, namely, block circulant systems, by exploiting the algebraic structure. The main theory of circulant dynamics considered in [8] is about circulant matrix.

Circulant matrices also play an important role in solving ordinary differential equations. By using a Strang-type block circulant preconditioner, Zhang et al. [9] speeded up the convergent rate of boundary-value methods. Delgado et al. [10] developed some techniques to obtain global hyperbolicity for a certain class of endomorphisms of $\left(R^{p}\right)^{n}$ with $p, n \geq 2$; this kind of endomorphisms was obtained from vectorial difference equations where the mapping defining these equations satisfies a circulant matrix condition. In [11], nonsymmetric, large, and sparse linear systems were solved by using the generalized minimal residual (GMRES) method; a circulant block preconditioner was proposed to speed up the convergence rate of the GMRES method. Wilde [12] developed a theory for the solution of ordinary and partial differential equations whose structure involves the algebra of circulants. He showed how the algebra of $2 \times 2$ circulants relates to the study of the harmonic oscillator, the CauchyRiemann equations, Laplace's equation, the Lorentz transformation, and the wave equation. And he used $n \times n$ circulants to suggest natural generalizations of these equations to higher dimensions. 
Circulant matrices have important applications in various disciplines including image processing [13-15], communications, signal processing [16], encoding, solving Toeplitz matrix problems, preconditioner, and solving least squares problems. They have been put on firm basis with the work of Davis [17] and Jiang and Zhou [18].

Some scholars have given various algorithms for the determinants and inverses of nonsingular circulant matrices $[17,18]$. Unfortunately, the computational complexity of these algorithms is exorbitant with the order of matrix increasing. However, some authors gave the explicit determinants and inverses of circulant and skew circulant involving some famous numbers. For example, Jaiswal evaluated some determinants of circulant whose elements are the generalized Fibonacci numbers [19]. Lind presented the determinants of circulant and skew circulant involving Fibonacci numbers [20]. Lin [21] gave the determinant of the Fibonacci-Lucas quasi-cyclic matrices. Shen considered circulant matrices with Fibonacci and Lucas numbers and presented their explicit determinants and inverses by constructing the transformation matrices [22]. Gao et al. [23] gave explicit determinants and inverses of skew circulant and skew left circulant matrices with Fibonacci and Lucas numbers. Jiang et al. $[24,25]$ considered the skew circulant and skew left circulant matrices with the $k$-Fibonacci numbers and the $k$-Lucas numbers and discussed the invertibility of these matrices and presented their determinant and inverse matrix by constructing the transformation matrices, respectively.

Recently, there are several papers on the norms of some special matrices. Solak [26] established the lower and upper bounds for the spectral norms of circulant matrices with classical Fibonacci and Lucas numbers entries. Ipek [27] investigated an improved estimation for spectral norms of these matrices. Shen and Cen [28] gave upper and lower bounds for the spectral norms of $r$-circulant matrices in the forms of $A=C_{r}\left(F_{0}, F_{1}, \ldots, F_{n-1}\right)$ and $B=C_{r}\left(L_{0}, L_{1}, \ldots, L_{n-1}\right)$, and they also obtained some bounds for the spectral norms of Kronecker and Hadamard products of matrix $A$ and matrix $B$. Akbulak and Bozkurt [29] found upper and lower bounds for the spectral norms of Toeplitz matrices such that $a_{i j} \equiv$ $F_{i-j}$ and $b_{i j} \equiv L_{i-j}$. The convergence in probability and the convergence in distribution of the spectral norm of scaled Toeplitz, circulant, reverse circulant, symmetric circulant, and a class of $k$-circulant matrices are discussed in [30].

Beginning with Mirsky [31], several authors [32-34] have obtained bounds for the spread of a matrix.

In this paper, by using the inverse factorization of polynomial of degree $n$, the explicit determinants of the circulant and left circulant matrix involving Tribonacci numbers and generalized Lucas numbers are expressed by utilizing only Tribonacci numbers and generalized Lucas numbers. Furthermore, the norms and some upper and lower bounds for the spread of these matrices are given, respectively.

The Tribonacci sequence $\left\{T_{n}\right\}$ and the generalized Lucas sequence $\left\{\mathbb{L}_{n}\right\}$ are defined by a third-order recurrence [3537]:

$$
\begin{array}{ll}
T_{n}=T_{n-1}+T_{n-2}+T_{n-3}, & n \geq 3, \\
\mathbb{L}_{n}=\mathbb{L}_{n-1}+\mathbb{L}_{n-2}+\mathbb{L}_{n-3}, & n \geq 3
\end{array}
$$

with the initial conditions $T_{0}=0, T_{1}=1, T_{2}=1$ and $\mathbb{L}_{0}=3$, $\mathbb{L}_{1}=1, \mathbb{L}_{2}=3$.

A few values of the sequences are given by the following table:

\begin{tabular}{c|cccccccccc}
$n$ & 0 & 1 & 2 & 3 & 4 & 5 & 6 & 7 & 8 & 9 \\
\hline$T_{n}$ & 0 & 1 & 1 & 2 & 4 & 7 & 13 & 24 & 44 & 81 \\
$\mathbb{L}_{n}$ & 3 & 1 & 3 & 7 & 11 & 21 & 39 & 71 & 131 & 241
\end{tabular}.

Note that $\tau_{i}$ are the roots of the characteristic equation $x^{3}-x^{2}-x-1=0$. Then, the Binet formulae of the sequences $\left\{T_{n}\right\}$ and $\left\{\mathbb{L}_{n}\right\}$ are

$$
\begin{gathered}
T_{n}=\Delta_{1} \tau_{1}^{n+1}+\Delta_{2} \tau_{2}^{n+1}+\Delta_{3} \tau_{3}^{n+1}, \\
\mathbb{L}_{n}=\tau_{1}^{n}+\tau_{2}^{n}+\tau_{3}^{n},
\end{gathered}
$$

where $\Delta_{i}=\prod_{j=1, j \neq i}^{3}\left(1 /\left(\tau_{i}-\tau_{j}\right)\right), i=1,2, \ldots, n$.

The relation between the roots and coefficient in the characteristic equations is

$$
\begin{gathered}
\tau_{1}+\tau_{2}+\tau_{3}=1, \\
\tau_{1} \tau_{2}+\tau_{1} \tau_{3}+\tau_{2} \tau_{3}=-1, \\
\tau_{1} \tau_{2} \tau_{3}=1 .
\end{gathered}
$$

Lemma 1. Several formulae concerning these sequences are listed as follows:

$$
\begin{gathered}
\sum_{j=1}^{n} T_{j}=\frac{T_{n}+T_{n+2}-1}{2}, \\
\sum_{j=1}^{n} \mathbb{L}_{j}=\frac{\mathbb{L}_{n}+\mathbb{L}_{n+2}}{2}-3, \\
\sum_{j=1}^{n} T_{j}^{2}=\frac{1+4 T_{n} T_{n+1}-\left(T_{n+1}-T_{n-1}\right)^{2}}{4}, \\
\sum_{j=1}^{n} \mathbb{L}_{j}^{2}=\frac{-\mathbb{L}_{n+1}^{2}-\mathbb{L}_{n-1}^{2}+\mathbb{L}_{2 n+3}+\mathbb{L}_{2 n-2}}{2}-2 .
\end{gathered}
$$

Proof. Firstly, we can find formula (7) in [37]. Secondly, we give the computation about the sum of the first $n$ numbers of those sequences.

According to the recurrence relations (1) and (4) and Binet formula of $\left\{T_{n}\right\}$, we can get

$$
\begin{aligned}
\sum_{j=1}^{n} T_{j} & =\sum_{j=1}^{n} \sum_{i=1}^{3} \Delta_{i} \tau_{i}^{j+1}=\sum_{i=1}^{3} \Delta_{i} \tau_{i}^{2} \frac{1-\tau_{i}^{n}}{1-\tau_{i}} \\
& =\frac{\sum_{i=1}^{3} \Delta_{i}\left(\tau_{i}+\tau_{i}^{3}-\tau_{i}^{n+1}-\tau_{i}^{n+3}\right)}{\left(1-\tau_{1}\right)\left(1-\tau_{2}\right)\left(1-\tau_{3}\right)} \\
& =\frac{T_{0}+T_{2}-T_{n}-T_{n+2}}{-2} \\
& =\frac{T_{n}+T_{n+2}-1}{2} .
\end{aligned}
$$


The assertions about the representation $\sum_{j=1}^{n} \mathbb{L}_{j}$ can be proved in the same way.

Finally, the quadratic sum of generalized Lucas sequences can be obtained as follows:

$$
\begin{aligned}
\sum_{j=1}^{n} \mathbb{L}_{j}^{2}= & \sum_{j=1}^{n}\left[\sum_{i=1}^{3} \tau_{i}^{j}\right]^{2} \\
= & \sum_{i=1}^{3} \sum_{j=1}^{n} \tau_{i}^{2 j}+2 \sum_{\substack{i, k=1 \\
i<k}}^{3} \sum_{j=1}^{n}\left(\tau_{i} \tau_{k}\right)^{j} \\
= & \frac{8-2 L_{2 n+1}-2 L_{2 n}}{-4} \\
& +2 \times\left[-\frac{L_{n+1}^{2}-L_{2(n+1)}}{4}-\frac{L_{n-1}^{2}-L_{2(n-1)}}{4}\right] \\
= & \frac{-\mathbb{L}_{n+1}^{2}-\mathbb{L}_{n-1}^{2}+\mathbb{L}_{2 n+3}+\mathbb{L}_{2 n-2}-2 .}{2}
\end{aligned}
$$

Hence, the proof is completed.

Definition 2 (see $[17,18]$ ). A circulant matrix $B \in M_{n}$, denoted by $\operatorname{Circ}\left(a_{1}, a_{2}, \ldots, a_{n}\right)$, is a matrix of the form

$$
B:=\left(\begin{array}{ccccc}
a_{1} & a_{2} & \ldots & a_{n-1} & a_{n} \\
a_{n} & a_{1} & a_{2} & \ldots & a_{n-1} \\
\vdots & a_{n} & a_{1} & \ddots & \vdots \\
a_{3} & \vdots & \ddots & \ddots & a_{2} \\
a_{2} & a_{3} & \ldots & a_{n} & a_{1}
\end{array}\right)_{n \times n} .
$$

Definition 3 (see $[17,18]$ ). A left circulant matrix $C \in M_{n}$, denoted by $\operatorname{LCirc}\left(a_{1}, a_{2}, \ldots, a_{n}\right)$, is a matrix of the form

$$
C:=\left(\begin{array}{ccccc}
a_{1} & a_{2} & a_{3} & \ldots & a_{n} \\
a_{2} & a_{3} & \ldots & a_{n} & a_{1} \\
a_{3} & . & . & . & \vdots \\
\vdots & a_{n} & a_{1} & \ldots & a_{n-2} \\
a_{n} & a_{1} & \ldots & a_{n-2} & a_{n-1}
\end{array}\right)_{n \times n} .
$$

Let $B=\operatorname{Circ}\left(a_{1}, a_{2}, \ldots, a_{n}\right)$ and $C=\operatorname{LCirc}\left(a_{1}, a_{2}, \ldots, a_{n}\right)$. By explicit computation, we find

$$
C=\Gamma B,
$$

where $\Gamma=\operatorname{Lcirc}(1,0,0, \ldots, 0)$.

Definition 4 (see [29]). Let $A=\left(a_{i j}\right)$ be an $n \times n$ matrix. The Euclidean (or Frobenius) norm, the spectral norm, the maximum column sum matrix norm, and the maximum row sum matrix norm of the matrix $A$ are, respectively,

$$
\begin{gathered}
\|A\|_{F}=\left(\sum_{i, j=1}^{n}\left|a_{i j}\right|^{2}\right)^{1 / 2}, \quad\|A\|_{2}=\left(\max _{1 \leq i \leq n} \lambda_{i}\left(A^{*} A\right)\right)^{1 / 2}, \\
\|A\|_{1}=\max _{1 \leq j \leq n} \sum_{i=1}^{n}\left|a_{i j}\right|, \quad\|A\|_{\infty}=\max _{1 \leq i \leq n} \sum_{j=1}^{n}\left|a_{i j}\right|,
\end{gathered}
$$

where $A^{*}$ denotes the conjugate transpose of $A$.
Definition 5 (see [32]). Let $A=\left(a_{i j}\right)$ be an $n \times n$ matrix with eigenvalues $\lambda_{i}, i=1,2, \ldots, n$. The spread of $A$ is defined as

$$
s(A)=\max _{i, j}\left|\lambda_{i}-\lambda_{j}\right|
$$

An upper bound for the spread due to Mirsky [31] states that

$$
s(A) \leq \sqrt{2\|A\|_{F}^{2}-\frac{2}{n}|\operatorname{tr} A|^{2}},
$$

where $\|A\|_{F}$ denotes the Frobenius norm of $A$ and $\operatorname{tr} A$ is the trace of $A$.

Lemma 6 (see [34]). If $A=\left(a_{i j}\right)$ is an $n \times n$ matrix, then

(i) if $A$ is real and normal, then $s(A) \geq(1 /(n-$ 1))| $\sum_{i \neq j} a_{i j} \mid$;

(ii) if $A$ is Hermitian, then $s(A) \geq 2 \max _{i \neq j}\left|a_{i j}\right|$.

Lemma 7 (see [30]). If $A$ is an $n \times n$ real symmetric or normal matrix, then we have $\|A\|_{2}=\max _{1 \leq i \leq n}\left|\lambda_{i}\right|$, where $\lambda_{i}(i=$ $1,2, \ldots, n)$ are the eigenvalues of $A$.

Lemma 8 (see $[17,18])$. Let $\varepsilon_{k}(k=1,2, \ldots, n)$ be the roots of the equation $x^{n}-1=0$. If $A=\operatorname{Circ}\left(a_{1}, a_{2}, \ldots, a_{n}\right)$, then the eigenvalues and determinant of $A$ are $\lambda_{k}=\sum_{j=1}^{n} a_{j} \varepsilon_{k}^{j-1}$ and $\operatorname{det} A=\prod_{k=1}^{n} \lambda_{k}=\prod_{k=1}^{n} \sum_{j=1}^{n} a_{j} \varepsilon_{k}^{j-1}$, respectively.

Lemma 9 (see [20]). Let $\varepsilon_{k}(k=1,2, \ldots, n)$ satisfy the equation $x^{n}-1=0$; then $\prod_{k=1}^{n}\left(y-\varepsilon_{k} z\right)=y^{n}-z^{n}, y, z \in \mathbf{C}$.

Lemma 10 (see [38]). Let $\theta_{k}=(2 k \pi / n), r_{k}=$ $\sum_{t=1}^{n} a_{t} \cos \left((t-1) \theta_{k}\right)$, and $s_{k}=\sum_{t=1}^{n} a_{t} \sin \left((t-1) \theta_{k}\right)$. If $N=\operatorname{LCirc}\left(a_{1}, a_{2}, \ldots, a_{n}\right)$, then the eigenvalues of $N$ are given by

$$
\lambda_{k}=-\lambda_{n-k}=\sqrt{r_{k}^{2}+s_{k}^{2}}, \quad 1 \leq k \leq\left\lfloor\frac{n-1}{2}\right\rfloor
$$

and $\lambda_{n}=\sum_{t=1}^{n} a_{t},\lfloor x\rfloor$ is the largest integer less than or equal to $x$. Note that, if $n$ is even, then $\lambda_{n / 2}=\sum_{t=1}^{n}(-1)^{t-1} a_{t}$.

\section{Determinant, Norms, and the Spread of Circulant and Left Circulant Matrices with Tribonacci Numbers}

Theorem 11. Let $A_{n}=\operatorname{Circ}\left(T_{1}, T_{2}, \ldots, T_{n}\right)$. Then the determinant of $A_{n}$ is

$$
\operatorname{det} A_{n}=\frac{\left(1-T_{n+1}\right)^{n}-\left(c_{1}^{n}+d_{1}^{n}\right)+\left(-T_{n}\right)^{n}}{\mathbb{L}_{-n}-\mathbb{L}_{n}},
$$


where

$$
\begin{gathered}
c_{1}=\frac{\left(T_{n+2}-T_{n+1}\right)+\mu_{1}}{2}, \\
d_{1}=\frac{\left(T_{n+2}-T_{n+1}\right)-\mu_{1}}{2}, \\
\mu_{1}=\sqrt{\left(T_{n+2}-T_{n+1}\right)^{2}-4 T_{n}\left(T_{n+1}-1\right)} .
\end{gathered}
$$

Proof. According to Lemma 8 and the Binet form of $\left\{T_{n}\right\}$, we obtain that the eigenvalues of $A_{n}$ are

$$
\begin{aligned}
& \lambda_{k}= \sum_{j=1}^{n} T_{j} \varepsilon_{k}^{j-1}=\sum_{j=1}^{n}\left(\sum_{i=1}^{3} \Delta_{i} \tau_{i}^{j+1}\right) \varepsilon_{k}^{j-1} \\
&= \sum_{i=1}^{3} \Delta_{i} \tau_{i}^{2}\left(\sum_{j=0}^{n-1} \tau_{i}^{j} \varepsilon_{k}^{j}\right) \\
&=\frac{1}{M}\left[\Delta_{1} \tau_{1}^{2}\left(1-\tau_{1}^{n}\right)\left(1-\tau_{2} \varepsilon_{k}\right)\left(1-\tau_{3} \varepsilon_{k}\right)\right. \\
& \quad+\Delta_{2} \tau_{2}^{2}\left(1-\tau_{2}^{n}\right)\left(1-\tau_{1} \varepsilon_{k}\right)\left(1-\tau_{3} \varepsilon_{k}\right) \\
&\left.\quad+\Delta_{3} \tau_{3}^{2}\left(1-\tau_{3}^{n}\right)\left(1-\tau_{1} \varepsilon_{k}\right)\left(1-\tau_{2} \varepsilon_{k}\right)\right] \\
&=\frac{1}{M}\left\{\begin{array}{l}
\Delta_{1} \tau_{1}^{2}\left(1-\tau_{1}^{n}\right)\left[1-\left(\tau_{2}+\tau_{3}\right) \varepsilon_{k}+\tau_{2} \tau_{3} \varepsilon_{k}^{2}\right] \\
\quad+\Delta_{2} \tau_{2}^{2}\left(1-\tau_{2}^{n}\right)\left[1-\left(\tau_{1}+\tau_{3}\right) \varepsilon_{k}+\tau_{1} \tau_{3} \varepsilon_{k}^{2}\right] \\
\left.\quad+\Delta_{3} \tau_{3}^{2}\left(1-\tau_{3}^{n}\right)\left[1-\left(\tau_{1}+\tau_{2}\right) \varepsilon_{k}+\tau_{1} \tau_{2} \varepsilon_{k}^{2}\right]\right\}
\end{array}\right.
\end{aligned}
$$

where $M=\prod_{i=1}^{3}\left(1-\tau_{i} \varepsilon_{k}\right)$ and $\varepsilon_{k}(k=1,2, \ldots, n)$ are the roots of $x^{n}-1=0$. From (4), we have

$$
\begin{aligned}
\lambda_{k} & =\frac{1}{M}\left[\left(-T_{n}\right) \varepsilon_{k}^{2}+\left(T_{n+1}-T_{n+2}\right) \varepsilon_{k}+\left(1-T_{n+1}\right)\right] \\
& =\frac{1}{M}\left(-T_{n}\right)\left(x_{1}-\varepsilon_{k}\right)\left(x_{2}-\varepsilon_{k}\right),
\end{aligned}
$$

where $x_{i}(i=1,2)$ are the roots of equation $\left(-T_{n}\right) \varepsilon_{k}^{2}+\left(T_{n+1}-\right.$ $\left.T_{n+2}\right) \varepsilon_{k}+\left(1-T_{n+1}\right)=0$. Applying Lemma 9, we have

$$
\begin{aligned}
\operatorname{det} A_{n} & =\frac{\left(-T_{n}\right)^{n}\left(x_{1}^{n}-1\right)\left(x_{2}^{n}-1\right)}{\left(1-\tau_{1}^{n}\right)\left(1-\tau_{2}^{n}\right)\left(1-\tau_{3}^{n}\right)} \\
& =\frac{\left(-T_{n}\right)^{n}\left[x_{1}^{n} x_{2}^{n}-\left(x_{1}^{n}+x_{2}^{n}\right)+1\right]}{1-\mathbb{L}_{n}+\mathbb{L}_{-n}-1} \\
& =\frac{\left(1-T_{n+1}\right)^{n}-\left(c_{1}^{n}+d_{1}^{n}\right)+\left(-T_{n}\right)^{n}}{\mathbb{L}_{-n}-\mathbb{L}_{n}},
\end{aligned}
$$

where

$$
\begin{gathered}
c_{1}=\frac{\left(T_{n+2}-T_{n+1}\right)+\mu_{1}}{2}, \\
d_{1}=\frac{\left(T_{n+2}-T_{n+1}\right)-\mu_{1}}{2}, \\
\mu_{1}=\sqrt{\left(T_{n+2}-T_{n+1}\right)^{2}-4 T_{n}\left(T_{n+1}-1\right)} .
\end{gathered}
$$

Theorem 12. Let $A_{n}=\operatorname{Circ}\left(T_{1}, T_{2}, \ldots, T_{n}\right)$. Then three kinds of norms of $A_{n}$ are given by

$$
\begin{gathered}
\left\|A_{n}\right\|_{1}=\left\|A_{n}\right\|_{\infty}=\frac{T_{n}+T_{n+2}-1}{2}, \\
\left\|A_{n}\right\|_{F}=\sqrt{n \cdot \frac{1+4 T_{n} T_{n+1}-\left(T_{n+1}-T_{n-1}\right)^{2}}{4}} .
\end{gathered}
$$

Proof. On the basis of the definitions of norms and (5) in Lemma 1, we have $\left\|A_{n}\right\|_{1}=\left\|A_{n}\right\|_{\infty}=\sum_{j=1}^{n} T_{j}=\left(\left(T_{n}+T_{n+2}-\right.\right.$ 1)/2).

According to the definition of norms and (7) in Lemma 1, we know that

$$
\begin{aligned}
\left\|A_{n}\right\|_{F}^{2} & =\sum_{i, j=1}^{n}\left|a_{i j}\right|^{2}=n \sum_{j=1}^{n} T_{j}^{2} \\
& =n \cdot \frac{1+4 T_{n} T_{n+1}-\left(T_{n+1}-T_{n-1}\right)^{2}}{4} ;
\end{aligned}
$$

hence, the Frobenius norm of $A_{n}$ is

$$
\left\|A_{n}\right\|_{F}=\sqrt{n \cdot \frac{1+4 T_{n} T_{n+1}-\left(T_{n+1}-T_{n-1}\right)^{2}}{4}} .
$$

Theorem 13. Let $A_{n}=\operatorname{Circ}\left(T_{1}, T_{2}, \ldots, T_{n}\right)$; then the spectral norm of $A_{n}$ is

$$
\left\|A_{n}\right\|_{2}=\frac{T_{n}+T_{n+2}-1}{2} .
$$

Proof. The modules of the eigenvalues of $A_{n}$ satisfy

$$
\begin{aligned}
\left|\lambda_{k}\left(A_{n}\right)\right| & =\left|\sum_{j=1}^{n} T_{j} \varepsilon_{k}^{j-1}\right| \\
& \leq \sum_{j=1}^{n}\left|T_{j}\right|\left|\varepsilon_{k}^{j-1}\right|=\sum_{j=1}^{n}\left|T_{j}\right|=\sum_{j=1}^{n} T_{j}, \\
A_{n} \cdot(1,1, \ldots, 1)^{T} & =\left(\sum_{j=1}^{n} T_{j}, \sum_{j=1}^{n} T_{j}, \ldots, \sum_{j=1}^{n} T_{j}\right)^{T} \\
& =\left[\sum_{j=1}^{n} T_{j}\right](1,1, \ldots, 1)^{T},
\end{aligned}
$$

which implies that $\sum_{j=1}^{n} T_{j}$ is an eigenvalue of $A_{n}$ and $\max _{1 \leq k \leq n}\left|\lambda_{k}\left(A_{n}\right)\right|=\sum_{j=1}^{n} T_{j}$. Hence, by Lemma 7 and equality (5) in Lemma 1 , we have $\|A\|_{2}=\max _{1 \leq k \leq n}\left|\lambda_{k}\left(A_{n}\right)\right|=$ $\sum_{j=1}^{n} T_{j}=\left(\left(T_{n}+T_{n+2}-1\right) / 2\right)$. 
Theorem 14. Let $A_{n}=\operatorname{Circ}\left(T_{1}, T_{2}, \ldots, T_{n}\right)$. Then the bounds for the spread of $A_{n}$ are

$$
\begin{gathered}
s\left(A_{n}\right) \geq \frac{n}{n-1} \cdot \frac{T_{n}+T_{n+2}-3}{2}, \\
s\left(A_{n}\right) \leq \sqrt{\frac{n\left[4 T_{n} T_{n+1}-\left(T_{n+1}-T_{n-1}\right)^{2}-3\right]}{2} .}
\end{gathered}
$$

Proof. The trace of $A_{n}$ is $\operatorname{tr} A_{n}=n T_{1}=n$ and $\sum_{i \neq j} a_{i j}=$ $n\left[\sum_{j=1}^{n} T_{j}-T_{1}\right]=n\left[\left(T_{n}+T_{n+2}-3\right) / 2\right]$. Since $A_{n}$ is a real and normal matrix, by using Lemma 6 , we can get $s\left(A_{n}\right) \geq$ $(1 /(n-1))\left|\sum_{i \neq j} a_{i j}\right|=(n /(n-1)) \cdot\left(\left(T_{n}+T_{n+2}-3\right) / 2\right)$.

Beside that, by Theorem 12, we have

$$
\begin{aligned}
2\left\|A_{n}\right\|_{F}^{2}-\frac{2}{n}\left|\operatorname{tr} A_{n}\right|^{2} \\
=2 n \cdot \frac{1+4 T_{n} T_{n+1}-\left(T_{n+1}-T_{n-1}\right)^{2}}{4}-\frac{2}{n} \cdot n^{2} \\
=n \cdot \frac{4 T_{n} T_{n+1}-\left(T_{n+1}-T_{n-1}\right)^{2}-3}{2} ;
\end{aligned}
$$

by (16), we obtain

$$
s\left(A_{n}\right) \leq \sqrt{n \cdot \frac{4 T_{n} T_{n+1}-\left(T_{n+1}-T_{n-1}\right)^{2}-3}{2}} .
$$

Theorem 15. Let $B_{n}=\operatorname{LCirc}\left(T_{1}, T_{2}, \ldots, T_{n}\right)$. Then the determinant of $B_{n}$ is

$$
\operatorname{det} B_{n}=\frac{\left(1-T_{n+1}\right)^{n}-\left(c_{1}^{n}+d_{1}^{n}\right)+\left(-T_{n}\right)^{n}}{\mathbb{L}_{-n}-\mathbb{L}_{n}}(-1)^{(n-1)(n-2) / 2} \text {. }
$$

Proof. Since

$$
\operatorname{det} \Gamma=(-1)^{(n-1)(n-2) / 2},
$$

the result can be derived from Theorem 11 and relation (13).

Theorem 16. Let $B_{n}=\operatorname{LCirc}\left(T_{1}, T_{2}, \ldots, T_{n}\right)$; then

$$
\begin{gathered}
\left\|B_{n}\right\|_{1}=\left\|B_{n}\right\|_{\infty}=\frac{T_{n}+T_{n+2}-1}{2}, \\
\left\|B_{n}\right\|_{F}=\sqrt{n \cdot \frac{1+4 T_{n} T_{n+1}-\left(T_{n+1}-T_{n-1}\right)^{2}}{4} .}
\end{gathered}
$$

Proof. By the definition of norms and formula (5) in Lemma 1, we know that $\left\|B_{n}\right\|_{1}=\left\|B_{n}\right\|_{\infty}=\sum_{j=1}^{n} T_{j}=\left(\left(T_{n}+\right.\right.$ $\left.T_{n+2}-1\right) / 2$ ).

According to Definition 4 and (7) in Lemma 1, we have

$$
\begin{aligned}
\left\|B_{n}\right\|_{F}^{2} & =\sum_{i, j=1}^{n}\left|b_{i j}\right|^{2}=n \sum_{j=1}^{n} T_{j}^{2} \\
& =n \cdot \frac{1+4 T_{n} T_{n+1}-\left(T_{n+1}-T_{n-1}\right)^{2}}{4} ;
\end{aligned}
$$

thus, the Frobenius norm of $B_{n}$ is

$$
\left\|B_{n}\right\|_{F}=\sqrt{n \cdot \frac{1+4 T_{n} T_{n+1}-\left(T_{n+1}-T_{n-1}\right)^{2}}{4}} .
$$

Theorem 17. Let $B_{n}=\operatorname{LCirc}\left(T_{1}, T_{2}, \ldots, T_{n}\right)$; then the spectral norm of $B_{n}$ is

$$
\left\|B_{n}\right\|_{2}=\frac{T_{n}+T_{n+2}-1}{2} .
$$

Proof. Obviously, the modules of the first $n-1$ eigenvalues of $B_{n}$ are

$$
\left|\lambda_{k}\right|=\left|\lambda_{n-k}\right|=\sqrt{r_{k}^{2}+s_{k}^{2}}, \quad 1 \leq k \leq\left\lfloor\frac{(n-1)}{2}\right\rfloor,
$$

and $\lambda_{n}=\sum_{j=1}^{n} T_{j}$ by Lemma 10. Since

$$
\begin{aligned}
& \sqrt{r_{k}^{2}+s_{k}^{2}} \\
& =\left|\sum_{j=1}^{n} T_{j} \cos \left((j-1) \theta_{k}\right)+i \sum_{j=1}^{n} T_{j} \sin \left((j-1) \theta_{k}\right)\right| \\
& =\left|\sum_{j=1}^{n} T_{j} e^{i(j-1) \theta_{k}}\right| \\
& \leq \sum_{j=1}^{n}\left|T_{j}\right|\left|e^{i(j-1) \theta_{k}}\right|=\sum_{j=1}^{n}\left|T_{j}\right|=\sum_{j=1}^{n} T_{j},
\end{aligned}
$$

we have $\left|\lambda_{k}\right|=\left|\lambda_{n-k}\right|=\sqrt{r_{k}^{2}+s_{k}^{2}} \leq \sum_{j=1}^{n} T_{j}$. Beside that, if $n$ is even, then

$$
\begin{aligned}
\left|\lambda_{n / 2}\right| & =\left|\sum_{j=1}^{n}(-1)^{j-1} T_{j}\right| \\
& \leq \sum_{j=1}^{n}\left|T_{j}\right|\left|(-1)^{j-1}\right|=\sum_{j=1}^{n}\left|T_{j}\right|=\sum_{j=1}^{n} T_{j} .
\end{aligned}
$$

In other words, for any $k=1,2, \ldots, n$, we have $\left|\lambda_{k}\right| \leq$ $\sum_{j=1}^{n} T_{j}=\lambda_{n}$, and $\lambda_{n}$ is an eigenvalue of $B_{n}$. So, $\max _{1 \leq k \leq n}\left|\lambda_{k}\left(B_{n}\right)\right|=\sum_{j=1}^{n} T_{j}$. Since $B_{n}$ is a real symmetric matrix, we can get $\left\|B_{n}\right\|_{2}=\max _{1 \leq k \leq n}\left|\lambda_{k}\left(B_{n}\right)\right|=\sum_{j=1}^{n} T_{j}=$ $\left(\left(T_{n}+T_{n+2}-1\right) / 2\right)$ by Lemma 7 and (5) in Lemma 1 .

Theorem 18. Let $B_{n}=\operatorname{LCirc}\left(T_{1}, T_{2}, \ldots, T_{n}\right)$; then the bounds for the spread of $B_{n}$ are

$$
s\left(B_{n}\right) \geq 2 T_{n},
$$

$$
\begin{aligned}
& s\left(B_{n}\right) \leq \sqrt{\mu_{2}-\frac{\left(T_{n}+T_{n+2}-1\right)^{2}}{2 n}} \quad(n \text { is odd }), \\
& s\left(B_{n}\right) \leq \sqrt{\mu_{2}-\frac{2}{n} \cdot\left(T_{n}+T_{n-1}\right)^{2}} \quad(n \text { is even }),
\end{aligned}
$$


where

$$
\mu_{2}=\frac{n\left[1+4 T_{n} T_{n+1}-\left(T_{n+1}-T_{n-1}\right)^{2}\right]}{2} .
$$

Proof. It follows from the elements in $B_{n}$ that $\max _{i \neq j}\left|b_{i j}\right|=$ $T_{n}$; since $B_{n}$ is a Hermitian matrix, so $s\left(B_{n}\right) \geq 2 \max _{i \neq j}\left|b_{i j}\right|=$ $2 T_{n}$.

If $n$ is odd, the trace of $B_{n}$ is $\operatorname{tr} B_{n}=\sum_{j=1}^{n} T_{j}=\left(\left(T_{n}+T_{n+2}-\right.\right.$ 1)/2). By using Theorem 16, we know that

$$
\begin{aligned}
2\left\|B_{n}\right\|_{F}^{2}-\frac{2}{n}\left|\operatorname{tr} B_{n}\right|^{2} \\
=2 n \cdot \frac{1+4 T_{n} T_{n+1}-\left(T_{n+1}-T_{n-1}\right)^{2}}{4} \\
-\frac{2}{n} \cdot\left[\frac{T_{n}+T_{n+2}-1}{2}\right]^{2} .
\end{aligned}
$$

If $n$ is even, the trace of $B_{n}$ is

$$
\begin{aligned}
\operatorname{tr} B_{n} & =2\left(T_{1}+T_{3}+T_{5}+\cdots+T_{n-1}\right) \\
& =2 \sum_{i=1}^{3} \Delta_{i} \frac{\tau_{i}^{2}\left(1-\tau_{i}^{2 \cdot(n / 2)}\right)}{1-\tau_{i}^{2}} \\
& =2 \cdot \frac{2\left(T_{n+1}-T_{n+2}\right)}{-4} \\
& =T_{n+2}-T_{n+1}=T_{n}+T_{n-1}
\end{aligned}
$$

by using Theorem 16, we have

$$
\begin{aligned}
2\left\|B_{n}\right\|_{F}^{2}-\frac{2}{n}\left|\operatorname{tr} B_{n}\right|^{2} \\
=2 n \cdot \frac{1+4 T_{n} T_{n+1}-\left(T_{n+1}-T_{n-1}\right)^{2}}{4} \\
\quad-\frac{2}{n} \cdot\left[T_{n}+T_{n-1}\right]^{2} .
\end{aligned}
$$

According to (16), the proof is completed.

\section{Determinant, Norms, and the Spread of Circulant and Left Circulant Matrices with Generalized Lucas Numbers}

Theorem 19. Let $C_{n}=\operatorname{Circ}\left(\mathbb{L}_{1}, \mathbb{L}_{2}, \ldots, \mathbb{L}_{n}\right)$. Then the determinant of $C_{n}$ is

$$
\operatorname{det} C_{n}=\frac{\left(1-\mathbb{L}_{n+1}\right)^{n}-\left(c_{2}^{n}+d_{2}^{n}\right)+\left(3-\mathbb{L}_{n}\right)^{n}}{\mathbb{L}_{-n}-\mathbb{L}_{n}},
$$

where

$$
\begin{gathered}
c_{2}=\frac{\left(\mathbb{L}_{n+2}-\mathbb{L}_{n+1}-2\right)+\mu_{3}}{2}, \\
d_{2}=\frac{\left(\mathbb{L}_{n+2}-\mathbb{L}_{n+1}-2\right)-\mu_{3}}{2}, \\
\mu_{3}=\sqrt{\left(\mathbb{L}_{n+2}-\mathbb{L}_{n+1}-2\right)^{2}-4\left(\mathbb{L}_{n}-3\right)\left(\mathbb{L}_{n+1}-1\right) .}
\end{gathered}
$$

Proof. By Lemma 8 and the Binet form of $\left\{\mathbb{L}_{n}\right\}$, the eigenvalues of $C_{n}$ are

$$
\begin{aligned}
& \lambda_{k}=\sum_{j=1}^{n} \mathbb{L}_{j} \varepsilon_{k}^{j-1}=\sum_{j=1}^{n} \sum_{i=1}^{3} \tau_{i}^{j} \varepsilon_{k}^{j-1} \\
& =\sum_{i=1}^{3} \tau_{i}\left(\sum_{j=0}^{n-1} \tau_{i}^{j} \varepsilon_{k}^{j}\right)=\sum_{i=1}^{3} \frac{\tau_{i}\left(1-\tau_{i}^{n}\right)}{1-\tau_{i} \varepsilon_{k}} \\
& =\frac{1}{M}\left[\tau_{1}\left(1-\tau_{1}^{n}\right)\left(1-\tau_{2} \varepsilon_{k}\right)\left(1-\tau_{3} \varepsilon_{k}\right)\right. \\
& +\tau_{2}\left(1-\tau_{2}^{n}\right)\left(1-\tau_{1} \varepsilon_{k}\right)\left(1-\tau_{3} \varepsilon_{k}\right) \\
& \left.+\tau_{3}\left(1-\tau_{3}^{n}\right)\left(1-\tau_{1} \varepsilon_{k}\right)\left(1-\tau_{2} \varepsilon_{k}\right)\right] \\
& =\frac{1}{M}\left\{\tau_{1}\left(1-\tau_{1}^{n}\right)\left[1-\left(\tau_{2}+\tau_{3}\right) \varepsilon_{k}+\tau_{2} \tau_{3} \varepsilon_{k}^{2}\right]\right. \\
& +\tau_{2}\left(1-\tau_{2}^{n}\right)\left[1-\left(\tau_{1}+\tau_{3}\right) \varepsilon_{k}+\tau_{1} \tau_{3} \varepsilon_{k}^{2}\right] \\
& \left.+\tau_{3}\left(1-\tau_{3}^{n}\right)\left[1-\left(\tau_{1}+\tau_{2}\right) \varepsilon_{k}+\tau_{1} \tau_{2} \varepsilon_{k}^{2}\right]\right\} ;
\end{aligned}
$$

according to (4), we have

$$
\begin{aligned}
\lambda_{k}= & \frac{1}{M} \sum_{i=1}^{3}\left(1-\tau_{i}^{n}\right)\left[\varepsilon_{k}^{2}-\tau_{i}\left(1-\tau_{i}\right) \varepsilon_{k}+\tau_{i}\right] \\
= & \frac{1}{M}\left[\left(3-\mathbb{L}_{n}\right) \varepsilon_{k}^{2}+\left(2+\mathbb{L}_{n+1}-\mathbb{L}_{n+2}\right) \varepsilon_{k}\right. \\
& \left.\quad+\left(1-\mathbb{L}_{n+1}\right)\right] \\
= & \frac{1}{M}\left(3-\mathbb{L}_{n}\right)\left(x_{3}-\varepsilon_{k}\right)\left(x_{4}-\varepsilon_{k}\right),
\end{aligned}
$$

where $x_{i}(i=3,4)$ are the roots of equation $\left(3-\mathbb{L}_{n}\right) \varepsilon_{k}^{2}+(2+$ $\left.\mathbb{L}_{n+1}-\mathbb{L}_{n+2}\right) \varepsilon_{k}+\left(1-\mathbb{L}_{n+1}\right)=0$.

According to Lemma 9, we have

$$
\begin{aligned}
\operatorname{det} C_{n} & =\frac{\left(3-\mathbb{L}_{n}\right)^{n}\left(x_{3}^{n}-1\right)\left(x_{4}^{n}-1\right)}{\left(1-\tau_{1}^{n}\right)\left(1-\tau_{2}^{n}\right)\left(1-\tau_{3}^{n}\right)} \\
& =\frac{\left(3-\mathbb{L}_{n}\right)^{n}\left[x_{3}^{n} x_{4}^{n}-\left(x_{3}^{n}+x_{4}^{n}\right)+1\right]}{1-\mathbb{L}_{n}+\mathbb{L}_{-n}-1} \\
& =\frac{\left(1-\mathbb{L}_{n+1}\right)^{n}-\left(c_{2}^{n}+d_{2}^{n}\right)+\left(3-\mathbb{L}_{n}\right)^{n}}{\mathbb{L}_{-n}-\mathbb{L}_{n}},
\end{aligned}
$$

where

$$
\begin{aligned}
& c_{2}=\frac{\left(\mathbb{L}_{n+2}-\mathbb{L}_{n+1}-2\right)+\mu_{3}}{2}, \\
& d_{2}=\frac{\left(\mathbb{L}_{n+2}-\mathbb{L}_{n+1}-2\right)-\mu_{3}}{2} \\
& \mu_{3}=\sqrt{\left(\mathbb{L}_{n+2}-\mathbb{L}_{n+1}-2\right)^{2}-4\left(\mathbb{L}_{n}-3\right)\left(\mathbb{L}_{n+1}-1\right)} .
\end{aligned}
$$


Theorem 20. Let $C_{n}=\operatorname{Circ}\left(\mathbb{L}_{1}, \mathbb{L}_{2}, \ldots, \mathbb{L}_{n}\right)$; then the norms of $C_{n}$ are

$$
\begin{gathered}
\left\|C_{n}\right\|_{1}=\left\|C_{n}\right\|_{\infty}=\frac{\mathbb{L}_{n}+\mathbb{L}_{n+2}}{2}-3, \\
\left\|C_{n}\right\|_{F}=\sqrt{n\left[\frac{-\mathbb{L}_{n+1}^{2}-\mathbb{L}_{n-1}^{2}+\mathbb{L}_{2 n+3}+\mathbb{L}_{2 n-2}}{2}-2\right] .}
\end{gathered}
$$

Proof. According to the definition of norms and formula (6) in Lemma 1, we obtain $\left\|C_{n}\right\|_{1}=\left\|C_{n}\right\|_{\infty}=\sum_{j=1}^{n} \mathbb{L}_{j}=\left(\left(\mathbb{L}_{n}+\right.\right.$ $\left.\left.\mathbb{L}_{n+2}\right) / 2\right)-3$.

According to the definition of norms and (8) in Lemma 1, we can get

$$
\begin{aligned}
\left\|C_{n}\right\|_{F}^{2} & =\sum_{i, j=1}^{n}\left|c_{i j}\right|^{2}=n\left[\sum_{j=1}^{n} \mathbb{L}_{j}^{2}\right] \\
& =n\left[\frac{-\mathbb{L}_{n+1}^{2}-\mathbb{L}_{n-1}^{2}+\mathbb{L}_{2 n+3}+\mathbb{L}_{2 n-2}}{2}-2\right] ;
\end{aligned}
$$

thus, the spectral norm of $C_{n}$ is

$$
\left\|C_{n}\right\|_{F}=\sqrt{n \cdot\left[\frac{-\mathbb{L}_{n+1}^{2}-\mathbb{\complement}_{n-1}^{2}+\mathbb{L}_{2 n+3}+\mathbb{L}_{2 n-2}}{2}-2\right]} .
$$

Theorem 21. Let $C_{n}=\operatorname{Circ}\left(\mathbb{L}_{1}, \mathbb{L}_{2}, \ldots, \mathbb{L}_{n}\right)$; then the spectral norm of $C_{n}$ is

$$
\left\|C_{n}\right\|_{2}=\frac{\mathbb{L}_{n}+\mathbb{L}_{n+2}}{2}-3
$$

Proof. The modules of the eigenvalues of $C_{n}$ satisfy

$$
\begin{aligned}
\left|\lambda_{k}\left(C_{n}\right)\right| & =\left|\sum_{j=1}^{n} \mathbb{L}_{j} \varepsilon_{k}^{j-1}\right| \\
& \leq \sum_{j=1}^{n}\left|\mathbb{L}_{j}\right|\left|\varepsilon_{k}^{j-1}\right|=\sum_{j=1}^{n}\left|\mathbb{L}_{j}\right|=\sum_{j=1}^{n} \mathbb{L}_{j}, \\
C_{n} \cdot(1,1, \ldots, 1)^{T} & =\left(\sum_{j=1}^{n} \mathbb{L}_{j}, \ldots, \sum_{j=1}^{n} \mathbb{L}_{j}\right)^{T} \\
& =\left[\sum_{j=1}^{n} \mathbb{L}_{j}\right](1,1, \ldots, 1)^{T},
\end{aligned}
$$

which means that $\sum_{j=1}^{n} \mathbb{L}_{j}$ is an eigenvalue of $C_{n}$, so $\max _{1 \leq k \leq n}\left|\lambda_{k}\left(C_{n}\right)\right|=\sum_{j=1}^{n} \mathbb{L}_{j}$. Hence, the spectral norm of $C_{n}$ is $\left\|C_{n}\right\|_{2}=\max _{1 \leq k \leq n}\left|\lambda_{k}\left(C_{n}\right)\right|=\sum_{j=1}^{n} \mathbb{L}_{j}=\left(\left(\mathbb{L}_{n}+\mathbb{L}_{n+2}\right) / 2\right)-3$ by Lemma 7 and formula (6) in Lemma 1.
Theorem 22. Let $C_{n}=\operatorname{Circ}\left(L_{1}, L_{2}, \ldots, L_{n}\right)$; then the bounds for the spread of $C_{n}$ are

$$
\begin{gathered}
s\left(C_{n}\right) \geq \frac{n}{n-1}\left[\frac{\mathbb{L}_{n}+\mathbb{L}_{n+2}}{2}-4\right], \\
s\left(C_{n}\right) \leq \sqrt{n\left(-\mathbb{L}_{n+1}^{2}-\mathbb{L}_{n-1}^{2}+\mathbb{L}_{2 n+3}+\mathbb{L}_{2 n-2}-6\right) .}
\end{gathered}
$$

Proof. The trace of $C_{n}$ is $\operatorname{tr} C_{n}=n \mathbb{L}_{1}=n$ and $\sum_{i \neq j} c_{i j}=$ $n\left[\sum_{j=1}^{n} \mathbb{L}_{j}-\mathbb{L}_{1}\right]=n\left[\left(\left(\mathbb{L}_{n}+\mathbb{L}_{n+2}\right) / 2\right)-4\right]$. Since $C_{n}$ is a real normal matrix, by Lemma 6 , we can get

$$
s\left(C_{n}\right) \geq \frac{1}{n-1}\left|\sum_{i \neq j} c_{i j}\right|=\frac{n}{n-1}\left[\frac{\mathbb{L}_{n}+\mathbb{L}_{n+2}}{2}-4\right] .
$$

Beside that, by Theorem 20, we have

$$
\begin{aligned}
2\left\|C_{n}\right\|_{F}^{2} & -\frac{2}{n}\left|\operatorname{tr} C_{n}\right|^{2} \\
= & 2 n \cdot\left[\frac{-\mathbb{L}_{n+1}^{2}-\mathbb{L}_{n-1}^{2}+\mathbb{L}_{2 n+3}+\mathbb{L}_{2 n-2}}{2}-2\right] \\
& -\frac{2}{n} \cdot n^{2} .
\end{aligned}
$$

By (16), the proof is completed.

Theorem 23. Let $D_{n}=\operatorname{LCirc}\left(\mathbb{L}_{1}, \mathbb{L}_{2}, \ldots, \mathbb{L}_{n}\right)$. Then

$$
\begin{aligned}
\operatorname{det} D_{n}= & \frac{\left(1-\mathbb{L}_{n+1}\right)^{n}-\left(c_{2}^{n}+d_{2}^{n}\right)+\left(3-\mathbb{L}_{n}\right)^{n}}{\mathbb{L}_{-n}-\mathbb{L}_{n}} \\
& \times(-1)^{(n-1)(n-2) / 2} .
\end{aligned}
$$

Proof. The conclusion can be proved by Theorem 19 and relation (13).

Theorem 24. Let $D_{n}=\operatorname{LCirc}\left(\mathbb{L}_{1}, \mathbb{L}_{2}, \ldots, \mathbb{L}_{n}\right)$; then the norms of $D_{n}$ are

$$
\begin{gathered}
\left\|D_{n}\right\|_{1}=\left\|D_{n}\right\|_{\infty}=\frac{\mathbb{L}_{n}+\mathbb{L}_{n+2}}{2}-3, \\
\left\|D_{n}\right\|_{F}=\sqrt{n \cdot\left[\frac{-\mathbb{L}_{n+1}^{2}-\mathbb{L}_{n-1}^{2}+\mathbb{L}_{2 n+3}+\mathbb{L}_{2 n-2}}{2}-2\right] .}
\end{gathered}
$$

Proof. According to the definition of norm and formula (6) in Lemma 1, we have $\left\|D_{n}\right\|_{1}=\left\|D_{n}\right\|_{\infty}=\sum_{j=1}^{n} \mathbb{L}_{j}=\left(\left(\mathbb{L}_{n}+\right.\right.$ $\left.\left.\mathbb{L}_{n+2}\right) / 2\right)-3$.

According to the definition of norm and (8) in Lemma 1, we can get

$$
\begin{aligned}
\left\|D_{n}\right\|_{F}^{2} & =\sum_{i, j=1}^{n}\left|d_{i j}\right|^{2}=n \sum_{j=1}^{n} \mathbb{L}_{j}^{2} \\
& =n\left[\frac{-\mathbb{L}_{n+1}^{2}-\mathbb{L}_{n-1}^{2}+\mathbb{L}_{2 n+3}+\mathbb{L}_{2 n-2}}{2}-2\right] ;
\end{aligned}
$$

thus, the Frobenius norm of $D_{n}$ is $\left\|D_{n}\right\|_{F}=$ $\sqrt{n\left[\left(\left(-\mathbb{L}_{n+1}^{2}-\mathbb{L}_{n-1}^{2}+\mathbb{L}_{2 n+3}+\mathbb{L}_{2 n-2}\right) / 2\right)-2\right]}$. 
Theorem 25. Let $D_{n}=\operatorname{LCirc}\left(\mathbb{L}_{1}, \mathbb{L}_{2}, \ldots, \mathbb{L}_{n}\right)$; then the spectral norm of $D_{n}$ is

$$
\left\|D_{n}\right\|_{2}=\frac{\mathbb{L}_{n}+\mathbb{\mathbb { L }}_{n+2}}{2}-3 .
$$

Proof. Obviously, the modules of the first $n-1$ eigenvalues of $D_{n}$ are

$$
\left|\lambda_{k}\right|=\left|\lambda_{n-k}\right|=\sqrt{r_{k}^{2}+s_{k}^{2}}, \quad 1 \leq k \leq\left\lfloor\frac{n-1}{2}\right\rfloor,
$$

and $\lambda_{n}=\sum_{j=1}^{n} \mathbb{L}_{j}$ by Lemma 10 . Since

$$
\begin{aligned}
& \sqrt{r_{k}^{2}+s_{k}^{2}} \\
& =\left|\sum_{j=1}^{n} \mathbb{L}_{j} \cos \left((j-1) \theta_{k}\right)+i \sum_{j=1}^{n} \mathbb{L}_{j} \sin \left((j-1) \theta_{k}\right)\right| \\
& =\left|\sum_{j=1}^{n} \mathbb{L}_{j} e^{i(j-1) \theta_{k}}\right| \\
& \leq \sum_{j=1}^{n}\left|\mathbb{L}_{j}\right|\left|e^{i(j-1) \theta_{k}}\right|=\sum_{j=1}^{n}\left|\mathbb{L}_{j}\right|=\sum_{j=1}^{n} \mathbb{L}_{j},
\end{aligned}
$$

we have $\left|\lambda_{k}\right|=\left|\lambda_{n-k}\right|=\sqrt{r_{k}^{2}+s_{k}^{2}} \leq \sum_{t=1}^{n} \mathbb{L}_{j}$. Beside that, if $n$ is even, then

$$
\begin{aligned}
\left|\lambda_{n / 2}\right| & =\left|\sum_{j=1}^{n}(-1)^{j-1} \mathbb{L}_{j}\right| \\
& \leq \sum_{j=1}^{n}\left|\mathbb{L}_{j}\right|\left|(-1)^{j-1}\right|=\sum_{j=1}^{n}\left|\mathbb{L}_{j}\right|=\sum_{j=1}^{n} \mathbb{L}_{j} .
\end{aligned}
$$

In other words, for any $k=1,2, \ldots, n$, we have $\left|\lambda_{k}\right| \leq$ $\sum_{j=1}^{n} \mathbb{L}_{j}=\lambda_{n}$, and $\lambda_{n}$ is an eigenvalue of $D_{n}$. So $\max _{1 \leq k \leq n}\left|\lambda_{k}\left(D_{n}\right)\right|=\sum_{j=1}^{n} \mathbb{L}_{j}$. Since $D_{n}$ is a real symmetric matrix, we can get $\left\|D_{n}\right\|_{2}=\max _{1 \leq k \leq n}\left|\lambda_{k}\left(D_{n}\right)\right|=\sum_{j=1}^{n} \mathbb{L}_{j}=$ $\left(\left(\mathbb{L}_{n}+\mathbb{L}_{n+2}\right) / 2\right)-3$ by Lemma 7 and (6) in Lemma 1 .

Theorem 26. Let $D_{n}=\operatorname{LCirc}\left(\mathbb{L}_{1}, \mathbb{L}_{2}, \ldots, \mathbb{L}_{n}\right)$; then the bounds for the spread of $D_{n}$ are

$$
\begin{gathered}
s\left(D_{n}\right) \geq 2 \mathbb{L}_{n}, \\
s\left(D_{n}\right) \leq \sqrt{n \cdot \mu_{4}-\frac{\left(\mathbb{L}_{n}+\mathbb{L}_{n+2}-6\right)^{2}}{2 n}}, \quad(n \text { is odd }), \\
s\left(D_{n}\right) \leq \sqrt{n \cdot \mu_{4}-\frac{2}{n} \cdot\left(\mathbb{L}_{n}+\mathbb{L}_{n-1}-2\right)^{2}}, \quad(n \text { is even }) . \\
\mu_{4}=-\mathbb{L}_{n+1}^{2}-\mathbb{L}_{n-1}^{2}+\mathbb{L}_{2 n+3}+\mathbb{L}_{2 n-2}-4 .
\end{gathered}
$$

Proof. From the elements in $D_{n}$, we know that $\max _{i \neq j}\left|d_{i j}\right|=$ $\mathbb{L}_{n}$; since $D_{n}$ is a Hermitian matrix, so $s\left(D_{n}\right) \geq 2 \max _{i \neq j}\left|d_{i j}\right|=$ $2 \mathbb{L}_{n}$.
If $n$ is odd, the trace of $D_{n}$ is $\operatorname{tr} D_{n}=\sum_{j=1}^{n} \mathbb{L}_{j}=\left(\left(\mathbb{Q}_{n}+\right.\right.$ $\left.\mathbb{L}_{n+2}\right) / 2$ ) - 3; by using Theorem 24 , we have

$$
\begin{aligned}
& 2\left\|D_{n}\right\|_{F}^{2}-\frac{2}{n}\left|\operatorname{tr} D_{n}\right|^{2}
\end{aligned}
$$

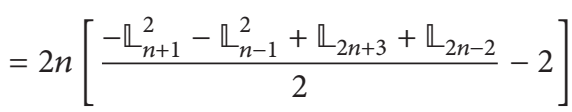

$$
\begin{aligned}
& -\frac{2}{n}\left[\frac{\mathbb{L}_{n}+\mathbb{\mathbb { L }}_{n+2}}{2}-3\right]^{2} \text {. }
\end{aligned}
$$

If $n$ is even,

$$
\begin{aligned}
\operatorname{tr} D_{n} & =2\left(\mathbb{L}_{1}+\mathbb{L}_{3}+\mathbb{L}_{5}+\cdots+\mathbb{L}_{n-1}\right) \\
& =2 \sum_{i=1}^{3} \frac{\tau_{i}\left(1-\tau_{i}^{2 \cdot(n / 2)}\right)}{1-\tau_{i}^{2}}=2 \cdot \frac{4+2\left(\mathbb{L}_{n+1}-\mathbb{L}_{n+2}\right)}{-4} \\
& =\mathbb{L}_{n+2}-\mathbb{L}_{n+1}-2=\mathbb{L}_{n}+\mathbb{L}_{n-1}-2 ;
\end{aligned}
$$

by using Theorem 24 , we have

$$
\begin{aligned}
& 2\left\|D_{n}\right\|_{F}^{2}-\frac{2}{n}\left|\operatorname{tr} D_{n}\right|^{2} \\
& =2 n\left[\frac{-\mathbb{L}_{n+1}^{2}-\mathbb{L}_{n-1}^{2}+\mathbb{L}_{2 n+3}+\mathbb{L}_{2 n-2}}{2}-2\right] \\
& -\frac{2}{n}\left[\mathbb{L}_{n}+\mathbb{E}_{n-1}-2\right]^{2} \text {. }
\end{aligned}
$$

According to (16), the conclusions are obtained.

\section{Conclusion}

The related problems of circulant matrix and some famous numbers are studied in this paper. We not only study basic properties of circulant matrix or famous numbers, respectively, but also explore the explicit determinant and the four kinds of norms and give the upper and lower bounds for the spread of circulant matrix and left circulant matrix involving Tribonacci numbers and generalized Lucas numbers. If we combine famous numbers with circulant matrix and left circulant matrix, a lot of good results would be obtained, and we wish the results could be useful in solving some differential equations.

\section{Conflict of Interests}

The authors declare that there is no conflict of interests regarding the publication of this paper.

\section{Acknowledgments}

The research was supported by the Development Project of Science and Technology of Shandong Province (Grant no. 2012GGX10115) and NSFC (Grant no.11301251) and the AMEP of Linyi University, China. 


\section{References}

[1] W. Chen, J. Lin, and C. S. Chen, "The method of fundamental solutions for solving exterior axisymmetric Helmholtz problems with high wave-number," Advances in Applied Mathematics and Mechanics, vol. 5, no. 4, pp. 477-493, 2013.

[2] S.-L. Lei and H.-W. Sun, "A circulant preconditioner for fractional diffusion equations," Journal of Computational Physics, vol. 242, pp. 715-725, 2013.

[3] B. Karasozen and G. Simsek, "Energy preserving integration of bi-Hamiltonian partial differential equations," Applied Mathematics Letters, vol. 26, no. 12, pp. 1125-1133, 2013.

[4] K. Otto, "Analysis of preconditioners for hyperbolic partial differential equations," SIAM Journal on Numerical Analysis, vol. 33, no. 6, pp. 2131-2165, 1996.

[5] E. W. Sachs and A. K. Strauss, "Efficient solution of a partial integro-differential equation in finance," Applied Numerical Mathematics, vol. 58, no. 11, pp. 1687-1703, 2008.

[6] A. E. Gilmour, "Circulant matrix methods for the numerical solution of partial differential equations by FFT convolutions," Applied Mathematical Modelling, vol. 12, no. 1, pp. 44-50, 1988.

[7] R. W. Brockett and J. L. Willems, "Discretized partial differential equations: examples of control systems defined on modules," Automatica, vol. 10, no. 5, pp. 507-515, 1974.

[8] S. E. Cohn and D. P. Dee, "Observability of discretized partial differential equations," SIAM Journal on Numerical Analysis, vol. 25, no. 3, pp. 586-617, 1988.

[9] C. Zhang, H. Chen, and L. Wang, "Strang-type preconditioners applied to ordinary and neutral differential-algebraic equations," Numerical Linear Algebra with Applications, vol. 18, no. 5, pp. 843-855, 2011.

[10] J. Delgado, N. Romero, A. Rovella, and F. Vilamajó, "Bounded solutions of quadratic circulant difference equations," Journal of Difference Equations and Applications, vol. 11, no. 10, pp. 897907, 2005.

[11] X.-Q. Jin, V.-K. Sin, and L.-L. Song, "Circulant-block preconditioners for solving ordinary differential equations," Applied Mathematics and Computation, vol. 140, no. 2-3, pp. 409-418, 2003.

[12] A. C. Wilde, "Differential equations involving circulant matrices," The Rocky Mountain Journal of Mathematics, vol. 13, no. 1, pp. 1-13, 1983.

[13] H.-J. Wittsack, A. M. Wohlschläger, E. K. Ritzl et al., "CTperfusion imaging of the human brain: advanced deconvolution analysis using circulant singular value decomposition," Computerized Medical Imaging and Graphics, vol. 32, no. 1, pp. 67-77, 2008.

[14] J. F. Henriques, R. Caseiro, P. Martins, and J. Batista, "Exploiting the circulant structure of tracking-by-detection with kernels," in Proceedings of the European Conference on Computer Vision (ECCV '12), pp. 1-14, 2012.

[15] X. Huang, G. Ye, and K.-W. Wong, "Chaotic image encryption algorithm based on circulant operation," Abstract and Applied Analysis, vol. 2013, Article ID 384067, 8 pages, 2013.

[16] M. Andrecut, "Applications of left circulant matrices in signal and image processing," Modern Physics Letters B, vol. 22, no. 4, pp. 231-241, 2008.

[17] P. J. Davis, Circulant Matrices, John Wiley \& Sons, New York, NY, USA, 1979.

[18] Z. L. Jiang and Z. X. Zhou, Circulant Matrices, Chengdu Technology University, Chengdu, China, 1999.
[19] D. V. Jaiswal, “On determinants involving generalized Fibonacci numbers," The Fibonacci Quarterly, vol. 7, pp. 319-330, 1969.

[20] D. A. Lind, "A Fibonacci circulant," The Fibonacci Quarterly, vol. 8, no. 5, pp. 449-455, 1970.

[21] D. Lin, "Fibonacci-Lucas quasi-cyclic matrices," The Fibonacci Quarterly, vol. 40, no. 3, pp. 280-286, 2002.

[22] S.-Q. Shen, J.-M. Cen, and Y. Hao, "On the determinants and inverses of circulant matrices with Fibonacci and Lucas numbers," Applied Mathematics and Computation, vol. 217, no. 23, pp. 9790-9797, 2011.

[23] Y. Gao, Z. L. Jiang, and Y. P. Gong, "On the determinants and inverses of skew circulant and skew left circulant matrices with Fibonacci and Lucas Numbers," WSEAS Transactions on Mathematics, vol. 12, no. 4, pp. 472-481, 2013.

[24] X. Y. Jiang, Y. Gao, and Z. L. Jiang, "Determinants and inverses of skew and skew left circulant matrices involving the kFibonacci numbers in communications-I," Far East Journal of Mathematical Sciences, vol. 76, no. 1, pp. 123-137, 2013.

[25] X. Y. Jiang, Y. Gao, and Z. L. Jiang, "Determinants and inverses of skew and skew left circulant matrices involving the kLucas numbers in communications-II," Far East Journal of Mathematical Sciences, vol. 78, no. 1, pp. 1-17, 2013.

[26] S. Solak, "On the norms of circulant matrices with the Fibonacci and Lucas numbers," Applied Mathematics and Computation, vol. 160, no. 1, pp. 125-132, 2005.

[27] A. Ipek, "On the spectral norms of circulant matrices with classical Fibonacci and Lucas numbers entries," Applied Mathematics and Computation, vol. 217, no. 12, pp. 6011-6012, 2011.

[28] S. Shen and J. Cen, "On the bounds for the norms of $r$ circulant matrices with the Fibonacci and Lucas numbers," Applied Mathematics and Computation, vol. 216, no. 10, pp. 2891-2897, 2010

[29] M. Akbulak and D. Bozkurt, "On the norms of Toeplitz matrices involving Fibonacci and Lucas numbers," Hacettepe Journal of Mathematics and Statistics, vol. 37, no. 2, pp. 89-95, 2008.

[30] A. Bose, R. S. Hazra, and K. Saha, "Spectral norm of circulanttype matrices," Journal of Theoretical Probability, vol. 24, no. 2, pp. 479-516, 2011.

[31] L. Mirsky, "The spread of a matrix," Mathematika, vol. 3, pp. 127130, 1956.

[32] R. Sharma and R. Kumar, "Remark on upper bounds for the spread of a matrix," Linear Algebra and its Applications, vol. 438, no. 11, pp. 4359-4362, 2013.

[33] J. Wu, P. Zhang, and W. Liao, "Upper bounds for the spread of a matrix," Linear Algebra and its Applications, vol. 437, no. 11, pp. 2813-2822, 2012.

[34] C. R. Johnson, R. Kumar, and H. Wolkowicz, "Lower bounds for the spread of a matrix," Linear Algebra and Its Applications, vol. 71, pp. 161-173, 1985.

[35] M. Elia, "Derived sequences, the Tribonacci recurrence and cubic forms," The Fibonacci Quarterly, vol. 39, no. 2, pp. 107-115, 2001.

[36] B. Balof, "Restricted tilings and bijections," Journal of Integer Sequences, vol. 15, Article ID 12.2.3, 2012.

[37] S. Rabinowitz, "Algorithmic manipulation of third-order linear recurrences," The Fibonacci Quarterly, vol. 34, no. 5, pp. 447464, 1996.

[38] A. Bose and J. Mitra, "Limiting spectral distribution of a special circulant," Statistics and Probability Letters, vol. 60, no. 1, pp. 111$120,2002$. 


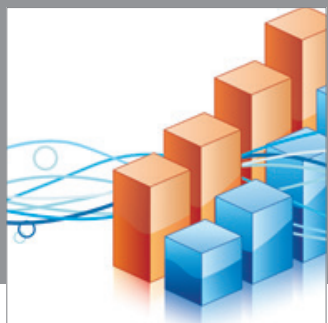

Advances in

Operations Research

mansans

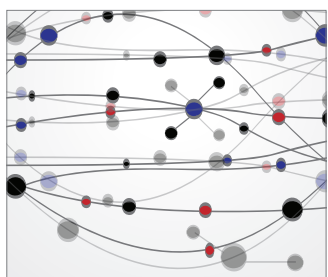

The Scientific World Journal
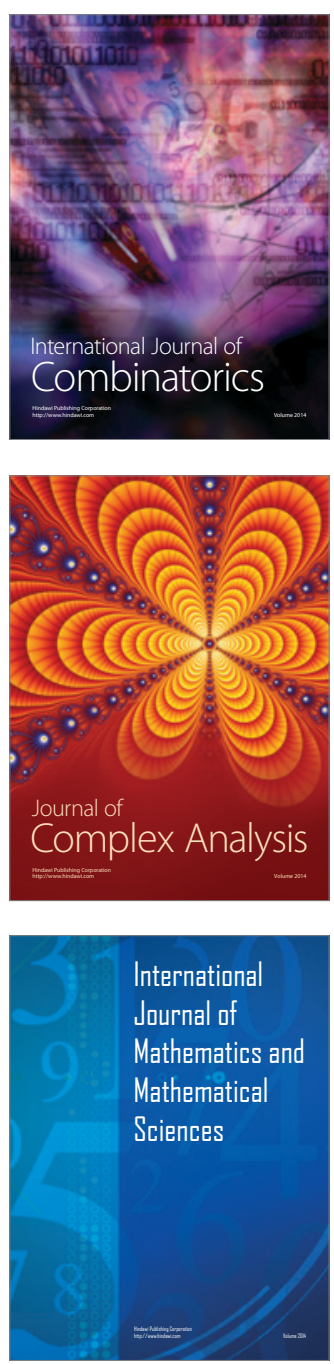
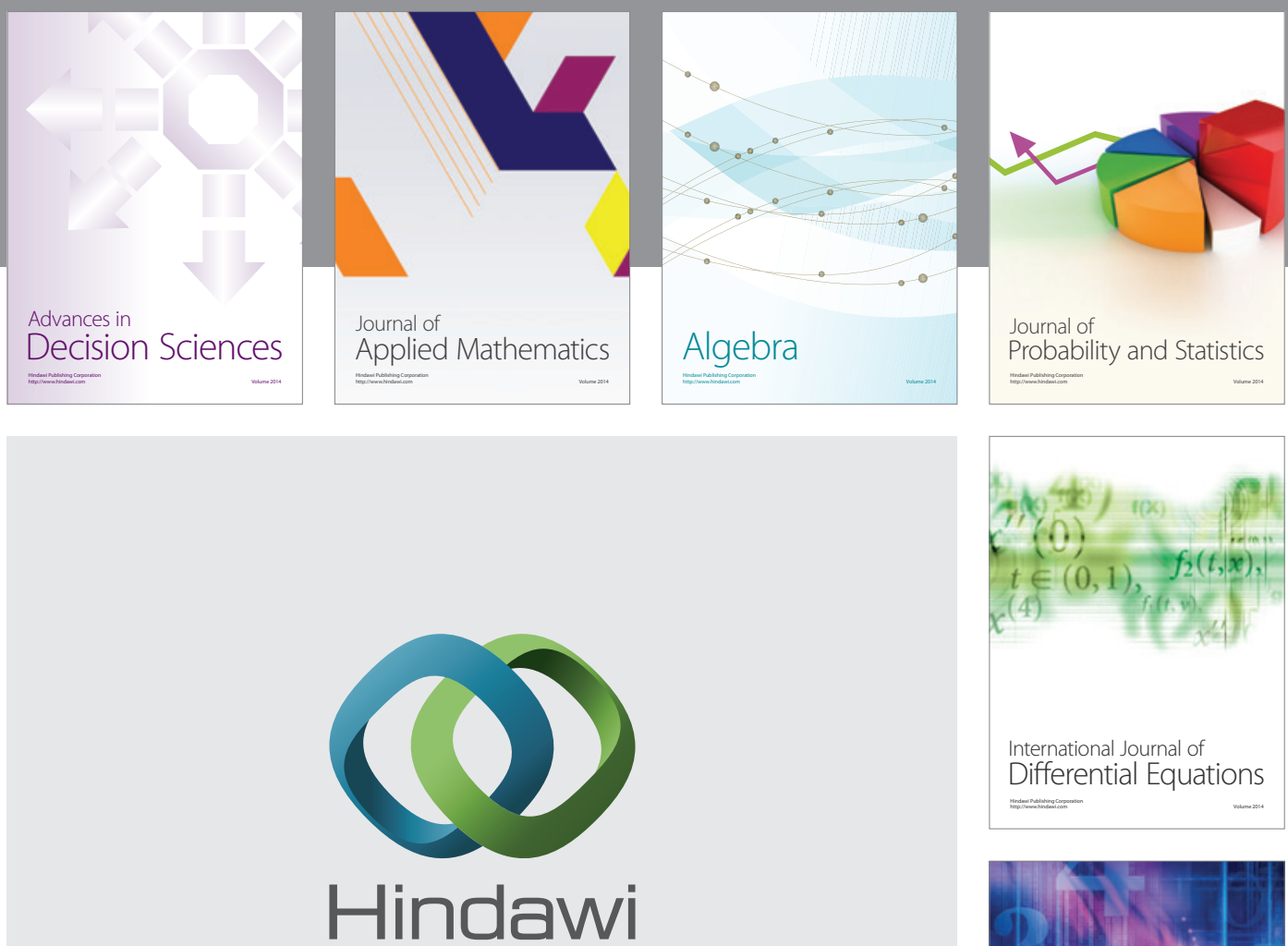

Submit your manuscripts at http://www.hindawi.com
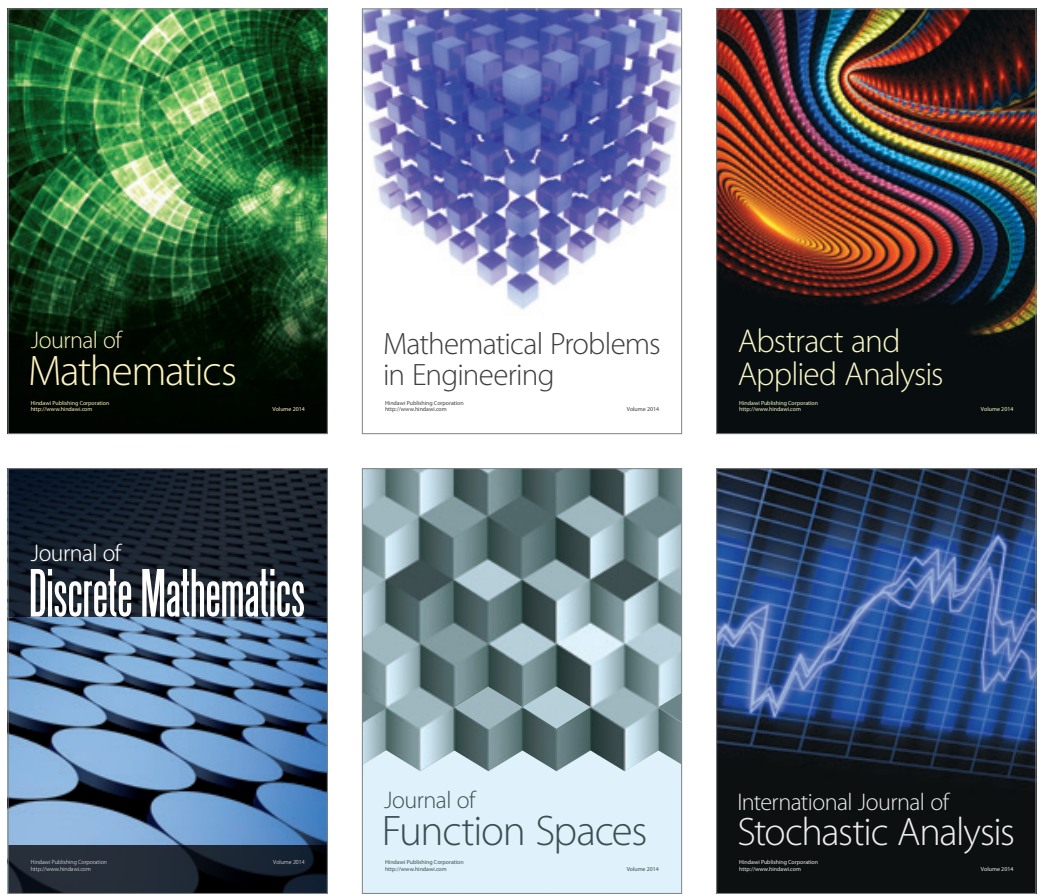

Journal of

Function Spaces

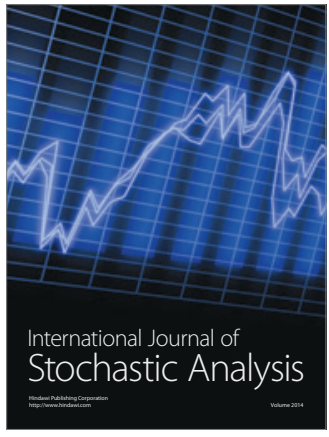

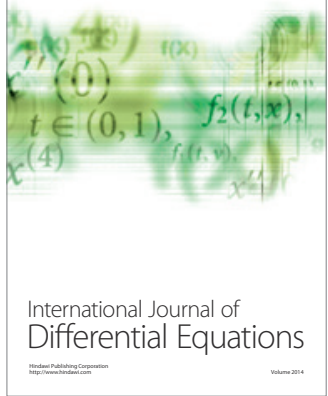
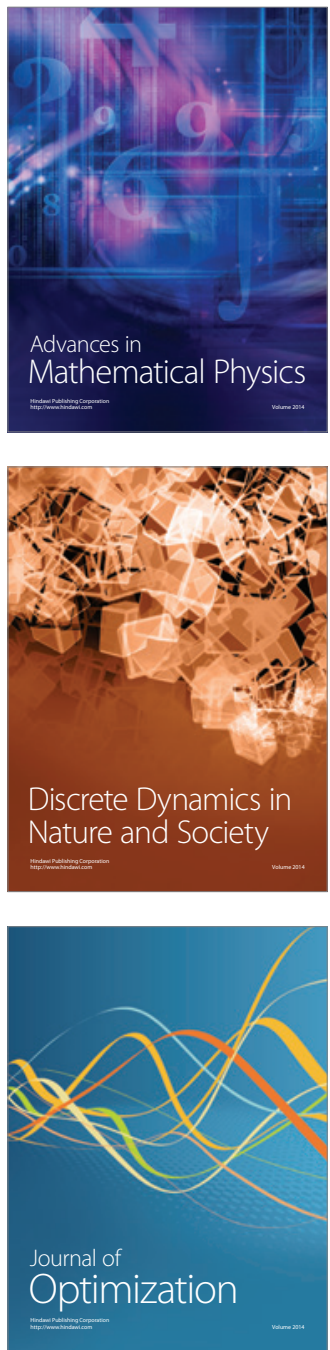